\title{
Vision-based Motion Control of a Biped Robot Using 2 DOF Gaze Control Structure
}

\author{
Shun Ushida and Koichiro Deguchi \\ Tohoku University \\ Japan
}

\section{Introduction}

Why "a biped robot"? Although, for this question, we can give various answers, no doubt we are motivated by existence of powerful controller of human brain. Human can exhibit intelligence and a flexible environmental adaptation ability. The concept of embodiment of robot, which is concerned with the human body and the function, allows us to give one of the questions from the point of view of intelligent mechanics. This concept suggests the necessity of the embodiment of humanoid robot in order to obtain an intelligence like human.

Recently, in a community of control engineering and control theory area, a control biology and a bio-mimetic control, which aim to elucidate and mimic a control mechanism for various organisms respectively, are interested by many researchers. In their investigations, a humanoid robot, in particular a motion control of a biped robot, has attracted attention for not only similarity of appearances between humanoid robots and humans but also the mechanism or intra-dynamics.

For the motion control of biped robots, we can not escape the fact that human motor control depends upon vision. Some recent researches on human motion control (Seara et al., 2002; Seara and Schmidt, 2004) have revealed that vision plays a major role in human posture control systems. Also, for the biped robot, vision is the most important sensory system for his posture and motion control.

On the other hand, the humanoid robots nowadays exploit a great variety of sensing technologies such as a angle and torque sensor of each joint, a gyroscope, a pressure sensor in a soul, a CCD camera, a microphone and a range finder. In fact, a humanoid robot shown in Fig. 1, which we use in our experiments, has above sensors in his body. Most of the sensorium of a robot is based on the so-called five senses of human. However, for a real humanoid robot, we have to assert that vision is one of underdeveloped sensors. This is mainly caused by two difficulties:

a. Poor Hardware Resources for vision: Hardware performance for robot vision on a biped robot is extremely restrictive due to limitations of the available hardware resources. For a small-sized humanoid robot, we can not use large-sized CCD camera, a heavyweight powerful actuator of the vision system.

b. An enormous amount of information in images: For image sequences from CCD camera, we have to extract useful information for robot control by using image processing. How ever, there is no systematic methodology of choosing a kind of visual information from an image. 


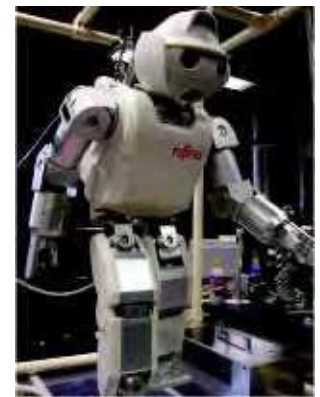

Figure 1. The humanoid robot called HOAP-3 made by Fujitsu Automation Corporation

The realization of vision of biped robots has many difficult problems to be tackled in aspects of both hardware and software (Afzulpurkar et al., 1996; Shibata and Schaal, 2001).

We clarify the structure and the nature of visual information processing system which biped robot should have. For this purpose, human eye structure and the eye movements by brain motor control are considered as an analogy for the humanoid robot system. We shall show that a gaze control mechanism like human is indispensable for vision system of biped robots. Actually, on the humanoid robot, we implemented the 2 DOF gaze control system which consists of the feedback and feedforward factor. Furthermore, we investigate considerable differences between human eyes and the vision of biped robot. This allows us to propose a new, simple and intuitively understandable criterion, called the range of gaze, for evaluating gaze control performance.

\section{A Vision System of Human}

In a human ocular system, we briefly review how one can obtain visual information for motor control efficiently.

\subsection{Structure of Ocular}

Detail of components of human ocular system has already been investigated from an anatomical point of view. In particular, on robotics, there are two important factors, eyeball driving system and image formation system.

Human eye movement is driven by 4 rectus muscles and 2 oblique muscles as shown in Fig. 2. 4 rectus muscles control a pan and tilt action of the eyeball. Since 2 muscles of rectus superior and inferior are not parallel on the gaze direction, these muscles cause not only tilt but also pan and slight roll. 2 oblique muscles cancel out this roll action. Hence, precise, quick and smooth eye movements of human can be achieved in superb combination with 6 muscles each other. A remarkable feature of these eye movements is that a motor unit of these muscles is greatly smaller than other muscle. In fact, 1 nerve fiber controls only 6 muscle fibers, whereas for a muscle for a finger movement 1 nerve fiber corresponds to 100 300 muscle ones. A maximum angular speed of eyeball is 300-500 [deg/s] and 1 action finishes within 30-50 [ms]. The eyeball is surrounded with an adipose tissue called corpus adiposum orbitae which plays a role as a rotational bearing.

Another important aspect of human ocular system on robotics is that the image formation system has also individual structure. An incident light from an external source through cornea is projected on retina after refraction by lens. Human eye has central visual field and 
peripheral visual field. In the former field, we can achieve high-quality and powerful perception of the object, but its scope is extremely narrow. Its angle is about 2.5 [deg]. The central visual field corresponds to a photoreceptor cell on retina called fovea centralis which enables us to realize keen vision. On the other hand, the latter is a visual field except for the central one and is used mainly as a motion detector.

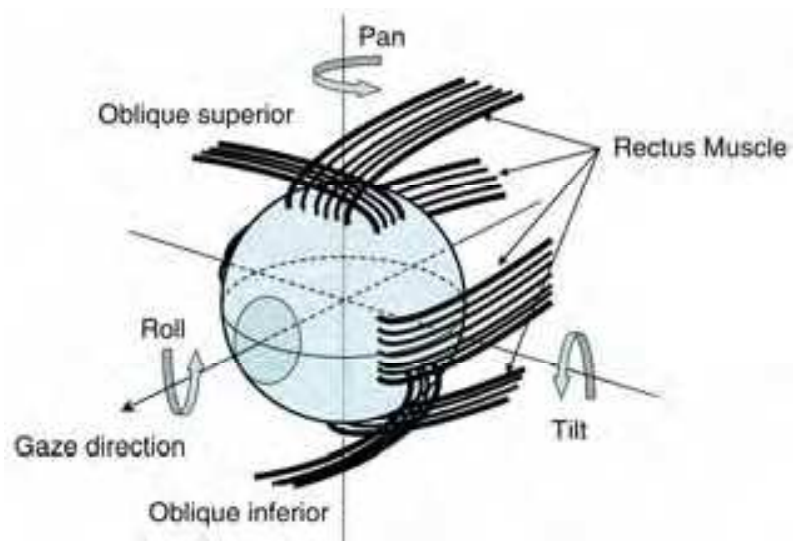

Figure 2. 6 Muscles for eye movement

\subsection{Some Ocular Movements}

The human eye movements are categorized by its function, a speed of eyeball, an organ caused the movement and so on. Here, we focus on voluntary eye movement and nonvoluntary one due to an intention of human. The voluntary eye movement consists of saccade and smooth pursuit movement, which are in order of the quickness of the speed. The saccade is stepwise response whose angular velocity is 200-500 [deg/s], whereas the other is smooth tracking movements with 20-120 [deg/s]. On the other hand, the non-voluntary eye movement is generated reflexively. The typical reflex action of an eyeball is called vestibuloocular reflex (VOR), which is directly due to a stimulus from a vestibular organ for a rotational movement of head. The VOR is related to human gaze control.

\subsection{A Constancy of a Position and Hierarchical Structure on Human Motor Control}

A model of control mechanism of eye movements have been studied since the middle of the 19th century from the various point of view of experimental psychology, medical science, physiology, engineering, robotics and so on. Due to complexity of human, however, it is difficult to capture the essential nature of eye movements and, therefore, the model in the individual theories, experiments and conjectures tend to be large-scale and complex. In this section, aiming at the applicability to robotics, we concentrate on two simple and intuitively understandable concepts called a constancy of a position in medical science, and a hierarchical structure on human motor control.

This constancy of a position means a constancy of visual world in our brain. For instance, when we are gazing an external object and moving ourselves by walking, running, bicycle and so on, we can feel the stationary visual world for a stationary scene whereas its image on our retina are moving due to a performance limitation of eye movements. This phenomenon has been conjectured by medical scientists that the transition of the image is 
compensated via a cancellation signal from the central nervous system which is stimulated by an other organ except for the retina.

The hierarchical structure on human motor control is related to the achievement of the constancy of a position which is realized by above eye movements such as a saccade and smooth pursuit movement. In this structure, we consider not only eye movements but also the constancy of a position based on eye movements, the perception of an object based on the constancy of a position, and the motor control based on the perception of an object, which form the hierarchical layers. This concept illustrated in Fig. 3, which was inspired by some results of experimental psychology (Recher and Oppenheim, 1955; Kinchla and Wolfe, 1979) and robotics (Westelius, 1995), is used in a subsequent section.

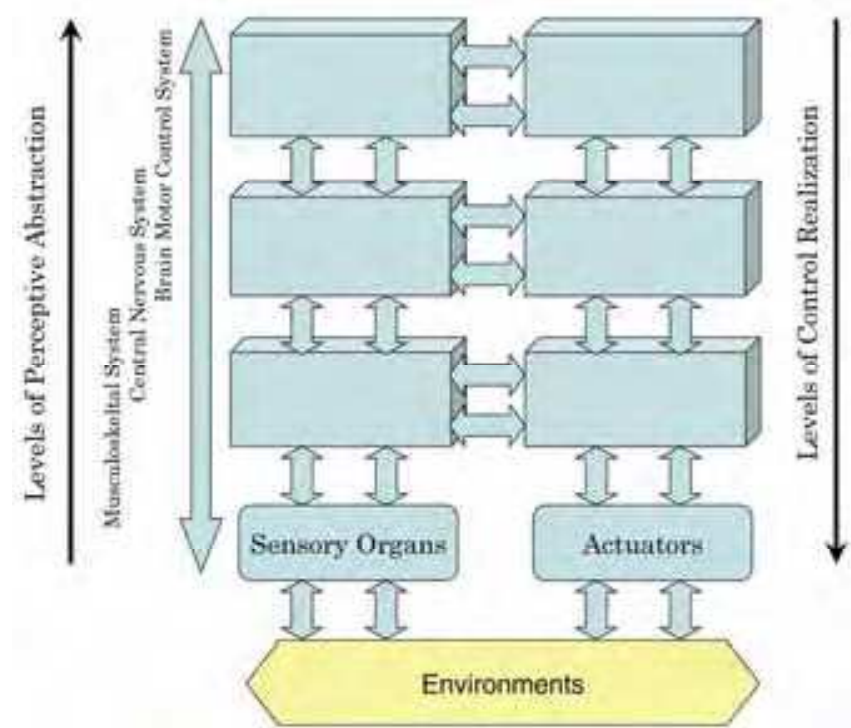

Figure 3. An integrated hierarchical structure on human motor control

\section{The Visual Information Processing System in a Biped Robot}

In this section, we discuss the visual information processing system which a biped robot should quip for vision-based motion control.

A biped robot consists of multiple joints which are precisely controlled by the computer. Since the repeatability for same controlled inputs is very high, the posture control of a biped robot seems to be simple apparently. However, the actual behavior is extremely complex due to a dynamically unstable structure of the biped robot and an interaction between the body and uncertain environments. This implies that the biped robot with a motion can not detect the position and posture himself. The vision enables us to get essential solution for this situation. If a biped robot has some sort of visual information processing system, it can observe an external fixation points as shown in Fig. 4. The vision-based biped robot can control the motion for the external fixation point. This does not mean the robot navigation system for a mobile robot. For unexpected motion error mentioned above, a vision can be used for primitive and basic motion control for autonomous biped robot. A use of the vision 
has some advantages. By using the fixed target, it can observe low-frequency postural change, which gyroscope sensors are hard to detect, and it is not affected by drift or accumulation of errors in the integration of sensor data (Seara et al., 2002).

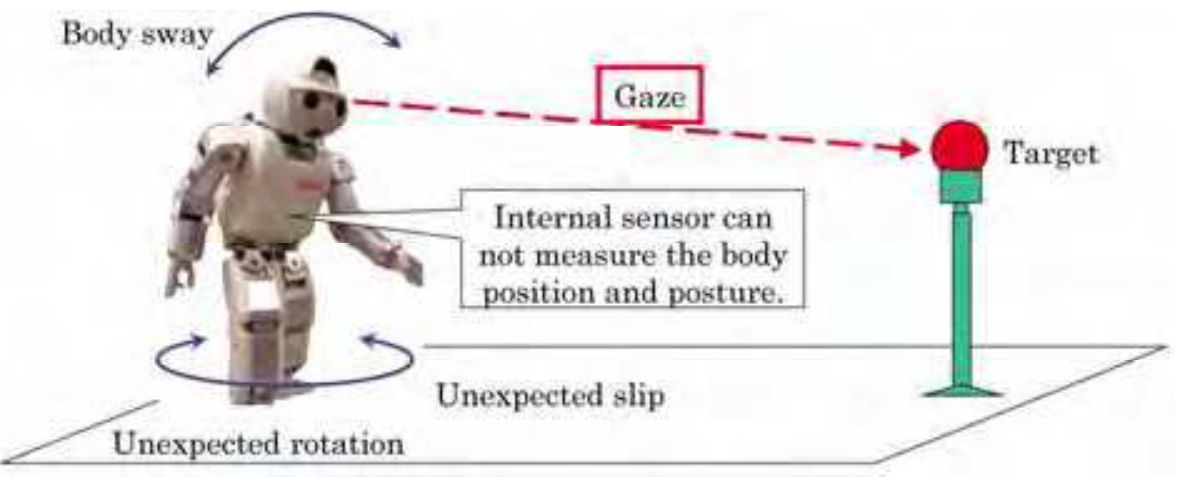

Figure 4 . The visual information processing system in the biped robot

\subsection{A Necessity of Gaze Control Mechanism}

A robot control with a vision, usually called a visual servoing, has studied by many researchers, e.g., (Kuniyoshi et al., 1995; Shibata and Schaal, 2001), who have dealt with a vision-based humanoid or robot arm fixed on a floor, a wheel-type mobile robot on which a CCD camera is installed and so on. However, a vision-based biped robot is absolutely different from others in the following points:

a. A vision is indispensable since the behavior of a biped robot depends on uncertain environment.

b. The vision system is usually located at a robot head, which swings violently.

c. In the motions of a biped robot such as walking and so on, the conflicts between legs and floor are repeatedly included.

d. The heavy visual devises and its processing system decrease the performance of motion significantly.

Under such a situation, the required features of the visual information processing system are the compactness and the enough performances, which, for example, are a wide view without skew, high resolution, high speed, high frame rate, effective real-time image processing algorism. However, it is difficult to satisfy these two features simultaneously. For instance, when the wide view is realized by using all-round camera or fish-eye lens, the skew of the obtained image is not avoidable. The more we require high resolution or frame rate, the more an amount of information of an image and movie increases. This yields a longer processing time. Furthermore, in general, high performance computer causes an increase of the weight of the system (Afzulpurkar et al, 1996; Ishikawa et al, 2002; Kurazume and Hirose, 2000; Shibata and Schaal, 2001).

In order to visual information for motor control on a biped robot without using such an inefficient architecture, introduction of gaze control mechanism is indispensable on biped robots. The human vision system in Sec. 2 gives effective suggestions for the situation of (a)(d). For an actuator which can pan and tilt the CCD camera for gazing, a concept of a constancy of a position and the hierarchical structure in Sec. 2.3 are useful. 
We can point out the following two important advantages of using a gaze control mechanism for a biped robot, the decrease of an amount of required information and the null method as measurement method.

For human as shown in Sec. 2.1, our perception with high-resolution is limited within narrow scope called central visual field. In fact, it is known that most of cortices of the visual area in the cerebrum are associated with information processing of the signals from the fovea centralis. In addition to this, an appropriate allocation of roles between the central visual field and the peripheral visual field can achieve a rapid and precise eye movements. In addition, it is known that the frame rate of human eye is at most or less than 30 [fps]. This fact implies that a use of image processing via brain in addition to gaze control can contribute to a decrease of an amount of visual information. On the other hand, in the CCDimage processing system on a biped robot, we can obtain same amount of information from each pixel of which an image consists. Actually, there is no difference of amount of information between a center part and a near part of the edge of an image. This fact can become an advantage and a disadvantage which depend on a required resolution, frame rate, capacity of a memory, a permissible processing time and so on, i.e., we can obtain high resolution image from same amount of information by using an appropriate zoom lens, and however time lag of capturing an image and its processing time can become the bottle neck of a control signal flow. Furthermore, by using appropriate image processing method we can realize a constancy of a position with low frame rate.

Another important nature of gaze control mechanism, the measurement of the posture of biped robot corresponds to a so-called null method in which a quantity to be measured is determined by making an adjustable quantity to be zero. When we consider the suitable resolution of an image, the capturing time and the processing time for a operating frequency of gaze control mechanism, this measurement method has some following advantages:

- the achievement and its improvement of a high accuracy of gaze control,

- the possibility of highly sensitive measurement for a motion of the robot, and

- the feasibility of constructing the responsive measurement system by a simple feedback loop structure.

The zero reference point corresponds to target to be gazed.

\subsection{A Concept of "the Range of Gaze"}

We introduce a notion of the range of gaze which is a simple and intuitively understandable criteria for evaluating gaze control performance.

Assume that an external fixation point, we call a target, is located at a center of an image at a time $t=t_{0}$. If a change of the view field of biped robot due to a motion happens at a time $t$, the target moves in the image as shown in Fig. 5(a). After a simple image processing, we can center the target in the view field as shown in Fig. $5(\mathrm{~b})$. The image area is characterized by the distances $d_{T}(t), d_{B}(t), d_{L}(t), d_{R}(t)$ in Fig. 5(b), and we denote this area as $A\left(d_{T}(t), d_{B}(t), d_{L}(t)\right.$, $\left.d_{R}(t)\right)$. If the target is not framed in the image, we assume that the area $A\left(d_{T}(t), d_{B}(t), d_{L}(t)\right.$, $\left.d_{R}(t)\right)$ vanishes, i.e., all distances are $d_{. .}(t)=0$. Then, we can define a notion of the range of gaze for a motion in a time sequences $t_{0}, \cdots t_{n}$ in the following:

$$
\bigcap_{t \in\left\{t_{0}, \cdots, t_{n}\right\}} A\left(d_{T}(t), d_{B}(t), d_{L}(t), d_{R}(t)\right) .
$$




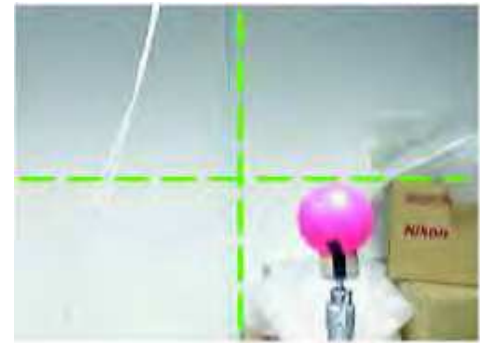

(a) The target position at time $t$

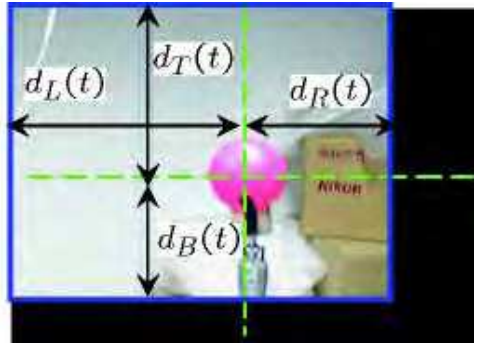

(b) After image shift

Figure 5. Concept of the range of gaze

A meaning of this concept is very clear. This enables us to validate a gaze control performance. For a motion, a wide range of gaze is desirable because this implies that an applicability of various type of image processing methods to this area. We can also achieve a perfect tracking of the target, which is a visual world we, human, are usually seeing. In this sense, obtaining the range of gaze from image sequences corresponds to a part of image processing in our brain.

\subsection{A Feedforward and Feedback Control Architecture on a Biped Robot}

In this section, we propose a new gaze control mechanism whose structural feature is a feedforward controller part with a database of robot motions (See also (Takizawa et al., 2004)). Our system as shown in Fig. 6 is the novel motion stabilization system of a biped robot with two degrees of freedom adaptive gaze control system.

This system consists of the fixed PID feedback controller and the adaptive feedforward controller $v t$ which is based on a scheduled motion and drives a pan of CCD camera. It was assumed that the scheduled motion for a walking is a sinusoid. However, this assumption is not necessarily true, which is shown in this paper. According to the control theory, in the 2 DOF control system, each role of the feedback and feedforward controller is mainly to stabilize the closed-loop systems and to improve the time responses, respectively. Therefore, an incorrect pan command generated by the feedforward controller adversely affects the behavior of CCD camera movements.

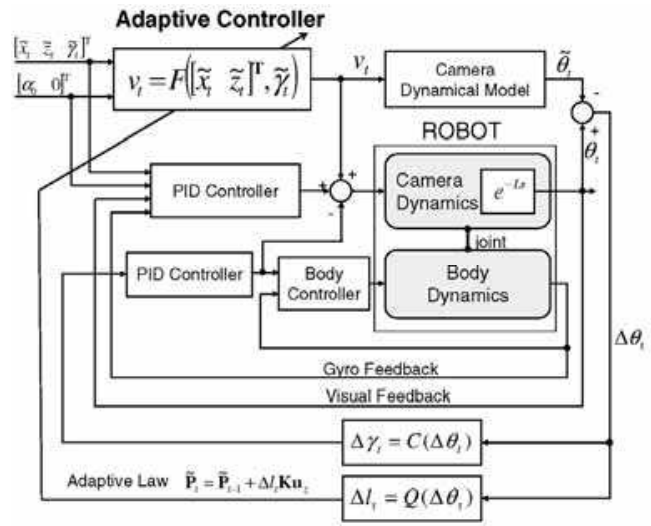

Figure 6. Block diagram of (Takizawa et al., 2004) 
To overcome this problem we propose a new feedforward controller which consists of a pan tilt command generator with a database of robot motions and a motion command generator as shown in Fig. 7. The data format in the database is a set of functions $f_{k}, k=1,2, \cdots \cdots, N$, where $N$ denotes the number of kinds of motions. Each $f_{k}$ depends on parameters which determine the motion, e.g., a period and a distance of a step and so on. The information of the parameters is provided by the motion command generator which drives all joints of the biped robot, except for the pan-tilt joint, to realize a fe-th scheduled motion. The pan tilt command generator and the motion command generator are synchronized by a reference signal from a timer.

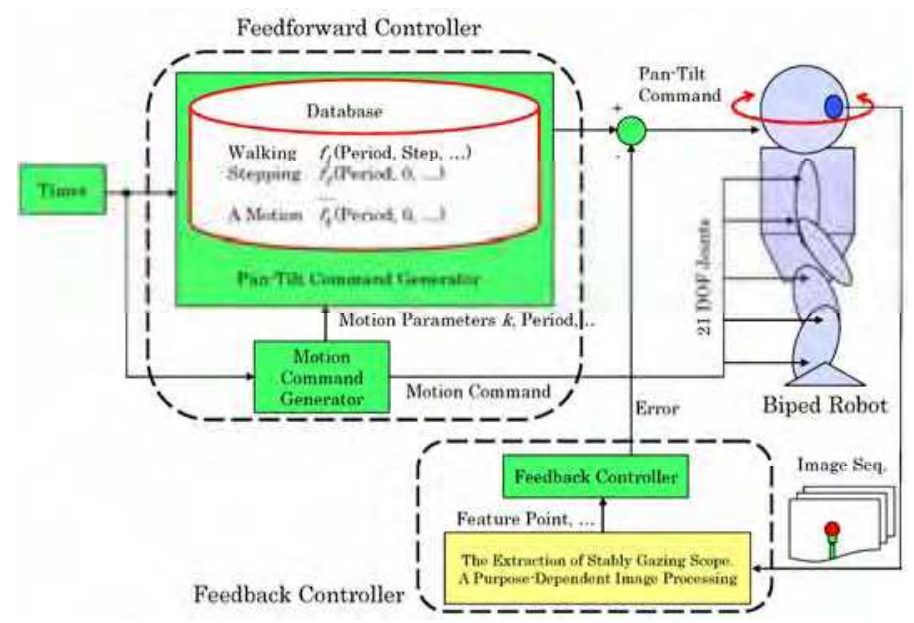

Figure 7. 2 DOF gaze control system with a database structure

As mentioned above, the function $f_{k}$ directly affects the performance of gaze control. In fact, for human motions which is quick, smooth and precise, it is known that a feedforward factor is predominantly used. Therefore, we can construct $f_{k}$ by using a precisely motiondependent signal which are measured by a high-speed external camera as a priori information. Hence, we can use a high performance camera system whose frame rate is approximately 20-50 times as a CCD camera of biped robot.

\subsection{Experiments}

We examined the feedforward control mechanism with a motion database and evaluate it via the range of gaze. To investigate an effect of the database to the gaze control system, we focused on the only feedforward part without any feedback loop. As mentioned in the preceding subsection, the feedforward factor determines predominantly the control performance. This is another reason why we dealt with only the feedforward part. The experimental setting is illustrated in Fig. 8. The biped robot is stepping/walking with the period $0.4[\mathrm{~Hz}]$. At first, in order to store the feedforward control inputs into the database, we measured these motions of biped robots, HOAP-1 and HOAP-3, by using a high-speed camera, nac Image Technology, Inc., HSV-500c3, 500 [fps]. Both HOAP-1 and HOAP-3 are made by Fujitsu Automation Cor. and HOAP-3, "3" means 3rd generation, is the successor robot of HOAP-1. Therefore, most of specs and performances of HOAP-3 surpass HOAP-1 
ones. This setting is illustrated in Fig. 9. By this measurement, we can know how much the head of a biped robot fluctuates during the motions. The results are shown in Fig. 10 and 11. In both case of HOAP-1 and HOAP-3, the difference of the motions appears as the remarkable difference of the fluctuation of the head. This fact supports our proposed gaze control mechanism, which includes motion-dependent pan-tilt command generator in the feedforward controller with database. Furthermore, the differences of HOAP-1 and HOAP-3 for same motions are also conspicuous, which implies that a desirable pan-tilt angle of a camera for gaze control extremely depends on an individual hardware of a biped robot. In our case, in particular, we observed that a structural difference of a pressure sensor in the soul of both robots causes the different behavior for both robots. Hence, as long as the hardware of a biped robot is developing nowadays, we must measure each motion and keep them in a database like our method.

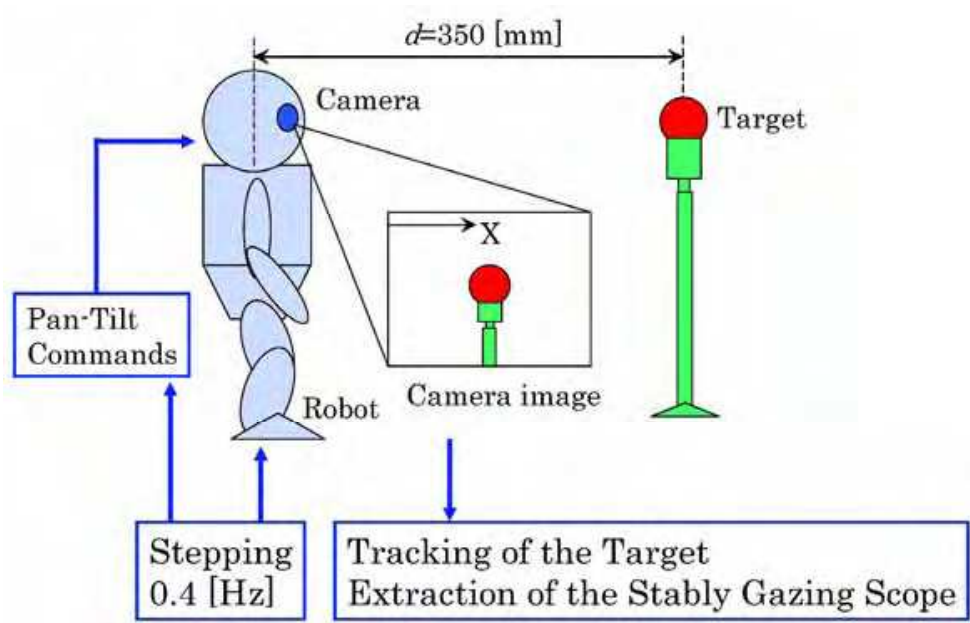

Figure 8 . The experimental setting of the gaze control during motions

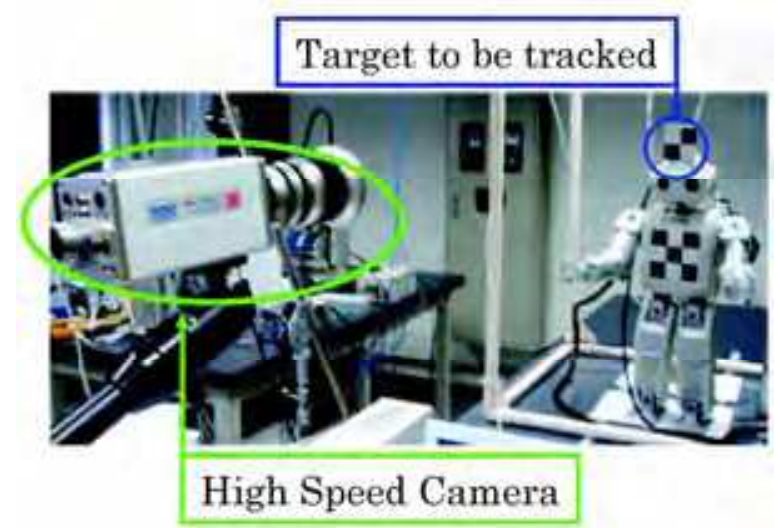

Figure 9. The high-speed camera and the biped robot, HOAP-3 
Fig. 12 and 13 show a time response of target displacement in an image and the range of gaze, respectively, during a step motion. The actual range of gaze is shown in Fig. 14. By using gaze control mechanism we can ensure a double and more area of the range of gaze.

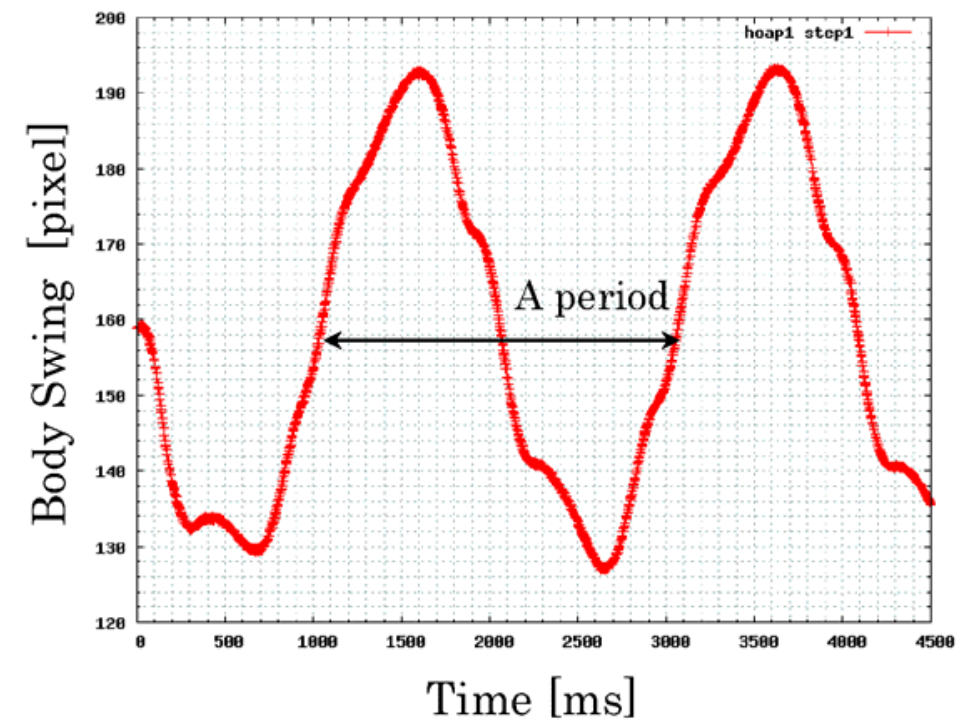

a) Stepping

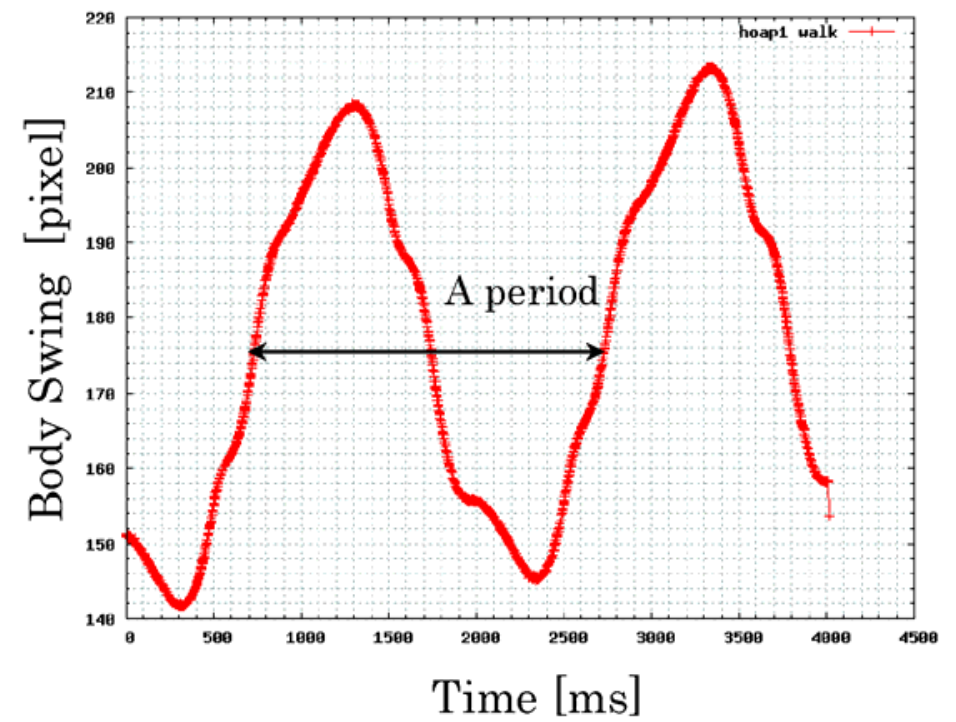

b) Walking

Figure 10. The results of the body swing during stepping/walking of HOAP-1 


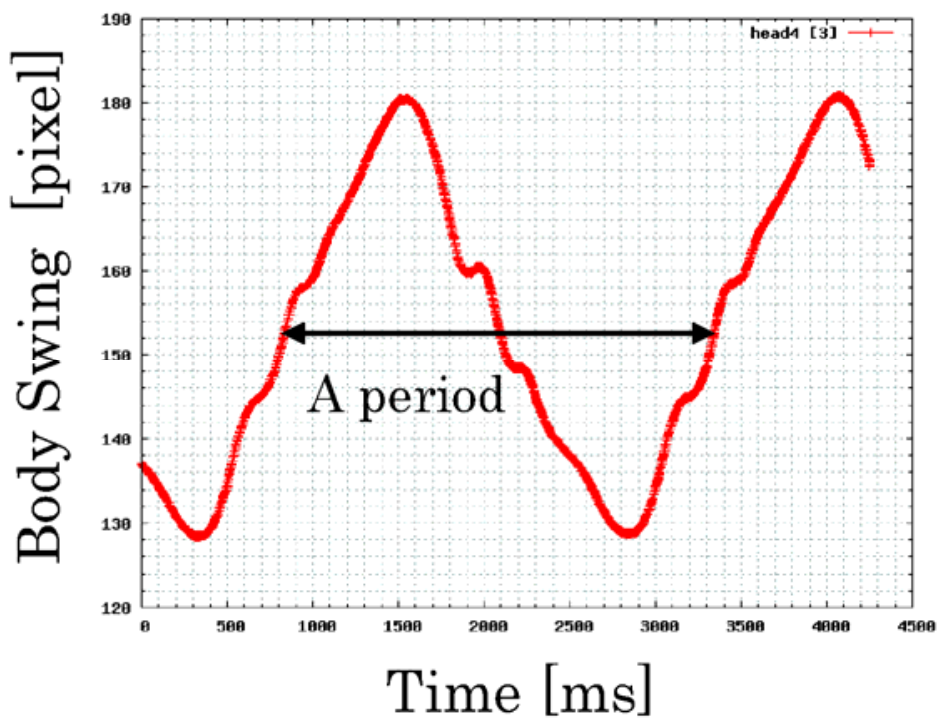

a) Stepping

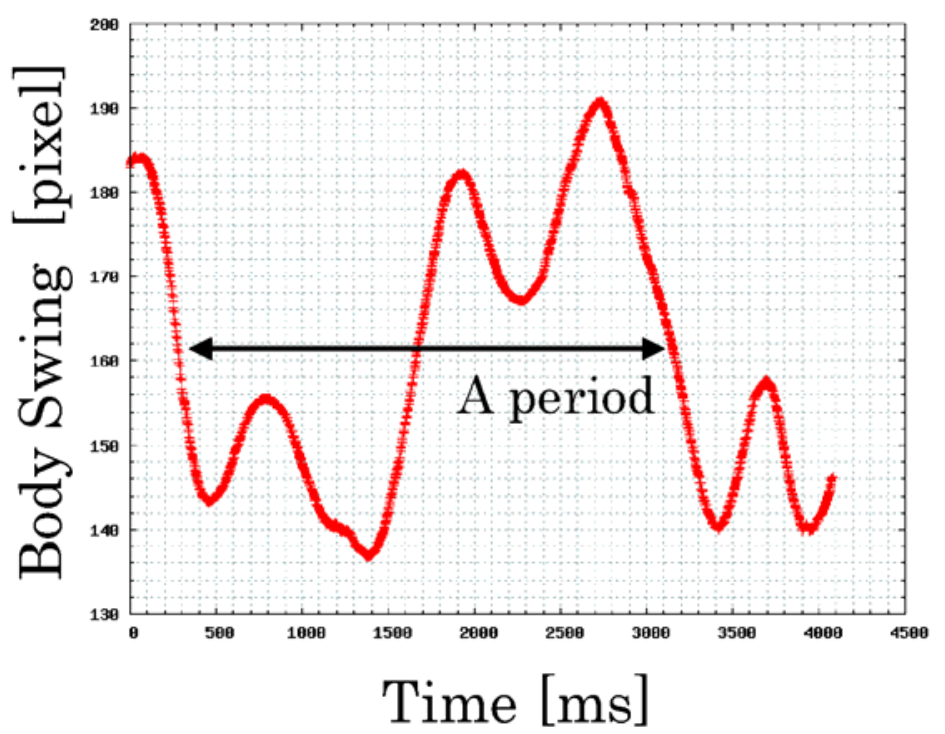

b) Walking

Figure 11. The results of the body swing during stepping/walking of HOAP-3 


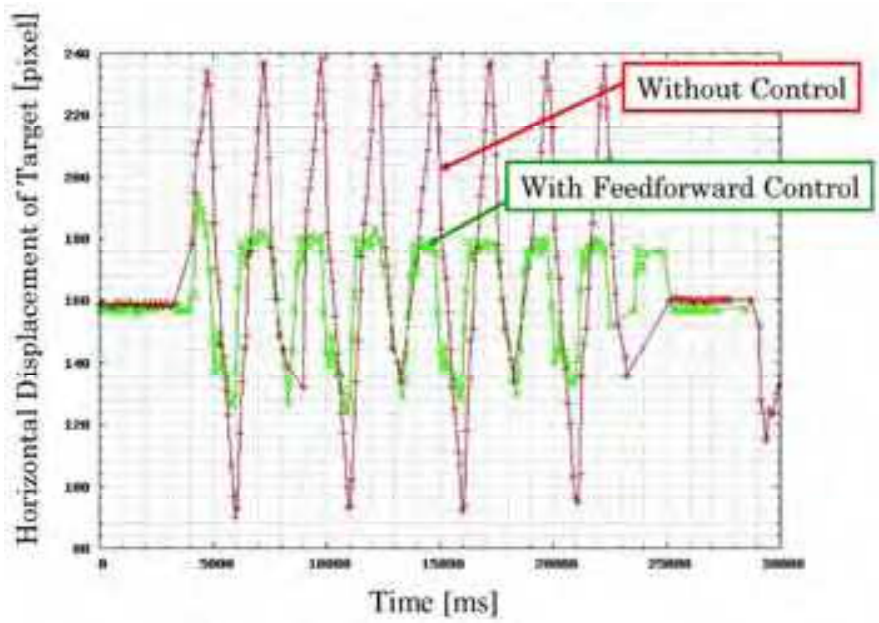

Figure 12. The result of a time response of target displacement for gaze control

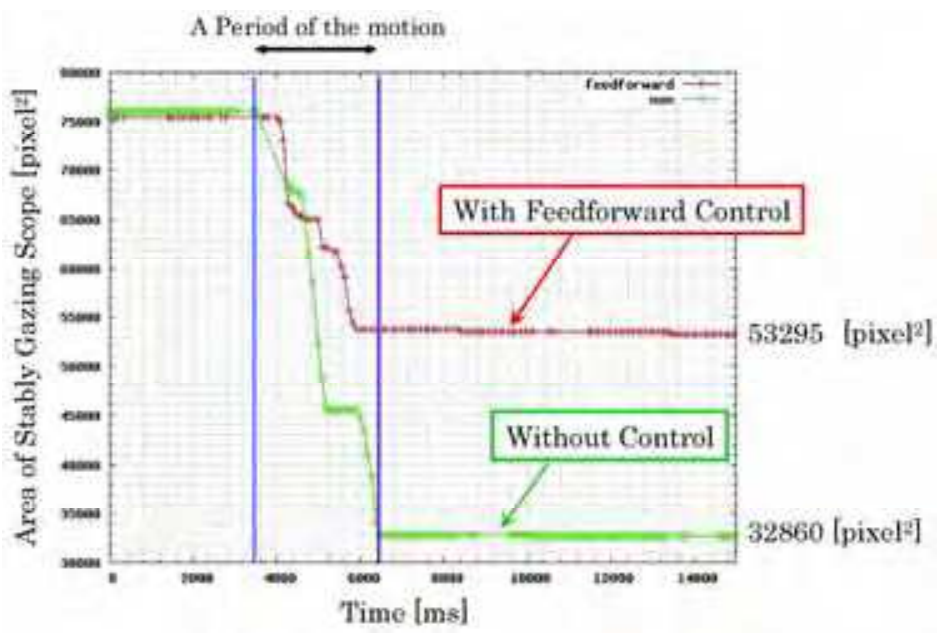

Figure 13. The result of a time response of the range of gaze for gaze control

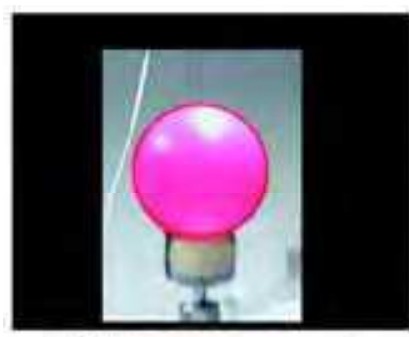

(a) Without any control

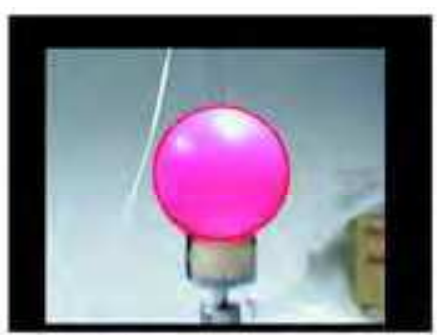

(b) With gaze control

Figure 14. The actual range of gaze via gaze control 


\section{Conclusion}

In this paper, we discussed both a necessity and importance of gaze control mechanism on vision-based motion control of biped robots. Human eye structure and the eye movements, and brain motor control through a central nervous system were considered as an analogy with robotics. In fact, human has powerful control mechanism with vision in order to realize a various type of motions against uncertain environments. As a result, we showed that gaze control mechanism and integrated hierarchical structure like human motor control are indispensable for a vision system of biped robots.

We can conclude that in order to overcome many difficulties of aspects of both a limitation of available hardware resources for a vision and a real-time image processing for motor control, we need not only gaze control mechanism but also use of the image processing technologies as shown in Fig. 15. We proposed a concept of the range of gaze and gaze control system with database structure. The use of both image processing and control theory allows us to realize an integrated hierarchical structure on the biped robot motion control as shown in Fig. 3. Furthermore, using the range of gaze, we can evaluate the gaze control performance from the point of view of the availability of visual information for robot motion control.

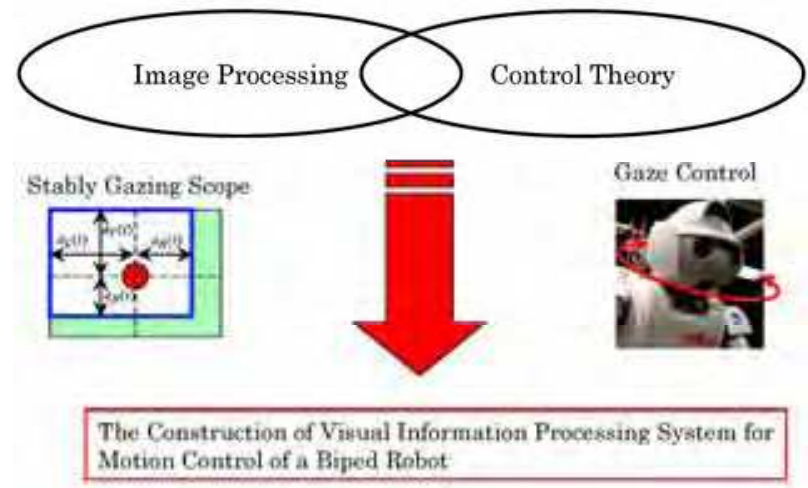

Figure 15. An integration of image processing and control theory on human motor control

\section{References}

Afzulpurkar, N.; Rougeaux, S.; Berthouze, L. \& Kuniyoshi, Y. (1996). A Gaze Based Approach for Visual Servoing, in Proc. of International Conference on Computational Engineering in System Application, pp. 570-575

Berthouze, L.; Rougeaux, S.; Chavand, R \& Kuniyoshi, Y. (1996). A Learning Stereo-Head Control System, in Proc. of World Automation Congress, International Symposium on Robotics and Manufacturing, In Robotics and Manufacturing: Recent trends in Research and Applications, Vol. 6, pp. 43-47

Demiris, J.; Rougeaux, S.; Hayes, G. M.; Berthouze, L. \& Kuniyoshi, Y. (1997). Deferred Imitation of Human Head Movements by an Active Stereo Vision Head, in Proc. of 6 th IEEE International Workshop on Robot and Human Communication, pp. 88-93 
Fukuoka, Y; Tanaka, K.; Ishida, A. \& Minamitani, H. (1999). Characteristics of Visually Feedback in Postural Control during Standing, IEEE Trans. Rehabilitation Engineering, Vol. 7, No. 4, pp. 427-434

Gutman, J.; Fukuchi, M. \& Fujita, M. (2004). Stair Climbing for Humanoid Robots Using Stereo Vision, in the Proc. of Int. Conf. on Intelligent Robots and Systems, pp. 1407-1413

Ishikawa, M.; Komuro, T.; Nakabo, Y. \& Namiki,A. (2002). The 1ms-Vision System and Its Application Examples, Workshop: Innovative Sensory-Motor Fusion Opens a New Robotic World (Organizers: Masatoshi Ishikawa, Makoto Kaneko), IEEE International Conference Robotics and Automation (ICRA 2002)

Jiang, Y.; Nagasaki, S. (2002). Health Evaluation Technology for Elders - Postural Sway Measurement and Postural Stability Evaluation during Upright Stance -, /. of Japanese Society for Non-destructive Inspection, Vol. 51, No. 9, pp. 567-572

Kinchla, R. A. \& Wolfe, J. M. (1979). The Order of Visual Processing: "Top-Down," "BottomUp," or "Middle-Out", Perception and Psychophysics, Vol. 25, pp. 225-231

Kuniyoshi, Y; Kita, N.; Rougeaux, S. \& Suehiro, T. (1995). Active Stereo Vision System with Foveated Wide Angle Lenses, in Proc. of Second Asian Conference on Computer Vision (ACCV95), pp. 359-363

Kurazume, R. \& Hirose, S. (2000). Development of image stabilization system for a remote operation of walking robots, in the Proc. IEEE International Conference on Robotics and Automation, pp. 1856-1861

Nakabo, Y; Fujikawa, N.; Mukai, T; Takeuchi, Y. \& Ohnishi, N. (2004). High-Speed and BioMimetic Control of a Stereo Head System, in Proc. of SICE Annual Conference, pp. 2371-2376

Navon, D. (1977). Forest before Tree: the Precedence of Global Features in Visual Perception, Cognitive Psychology, Vol. 9, pp. 353-383

Rescher, N. E Oppenheim, P. (1955). Logical Analysis of Gestalt Concepts, British Journal for the Philosophy of Science, Vol. 6, pp. 89-106

Seara, J.; Strobl, K. \& Schmidt, G. (2002). Information Management for Gaze Control in Vision Guided Biped Walking, in Proc. of Int. Conf. of Intelligence Robots and Systems, pp. 31-36

Seara, J. \& Schmidt, G. (2004). Intelligent Gaze Control for Vision-Guided Humanoid Walking: Methodological Aspects, Robotics and Autonomous Systems, Vol. 48, pp. 231-248

Shibata, T. \& Schaal, S. (2001). Biomimetic Gaze Stabilization based on Feedback-ErrorLearning with Nonparametric Regression Networks, Neural Networks, Vol. 14, pp. 201-216

Takizawa, S.; Ushida, S.; Okatani, T. \& Deguchi, K. (2001). 2DOF Motion Stabilization of Biped Robot by Gaze Control Strategy, in Proc. of IEEE/RSJ International Conference on Intelligent Robots and Systems (IROS2004), pp. 3809-3814

Ushida, S.; Terashita, J. \& Kimura, H. (2004). Switching Structural Biomechanical Model of Multisensory Integration during Human Quiet Standing, in Proc. of 43rd IEEE Conf. on Decision and Control (CDC2004), pp. 959-965

Westelius, C. J. (1995). Focus of Attention and Gaze Control for Robot Vision, Ph. D Thesis, Linkoping University, Sweden, Dissertation No. 379 


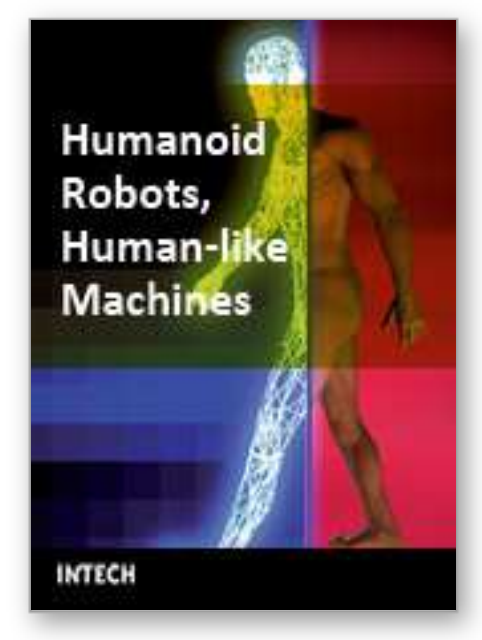

\author{
Humanoid Robots, Human-like Machines \\ Edited by Matthias Hackel
}

ISBN 978-3-902613-07-3

Hard cover, 642 pages

Publisher I-Tech Education and Publishing

Published online 01, June, 2007

Published in print edition June, 2007

In this book the variety of humanoid robotic research can be obtained. This book is divided in four parts: Hardware Development: Components and Systems, Biped Motion: Walking, Running and Self-orientation, Sensing the Environment: Acquisition, Data Processing and Control and Mind Organisation: Learning and Interaction. The first part of the book deals with remarkable hardware developments, whereby complete humanoid robotic systems are as well described as partial solutions. In the second part diverse results around the biped motion of humanoid robots are presented. The autonomous, efficient and adaptive two-legged walking is one of the main challenge in humanoid robotics. The two-legged walking will enable humanoid robots to enter our environment without rearrangement. Developments in the field of visual sensors, data acquisition, processing and control are to be observed in third part of the book. In the fourth part some "mind building" and communication technologies are presented.

\title{
How to reference
}

In order to correctly reference this scholarly work, feel free to copy and paste the following:

Shun Ushida and Koichiro Deguchi (2007). Vision-based Motion Control of a Biped Robot Using 2 DOF Gaze Control Structure, Humanoid Robots, Human-like Machines, Matthias Hackel (Ed.), ISBN: 978-3-902613-07-3, InTech, Available from: http://www.intechopen.com/books/humanoid_robots_human_like_machines/visionbased_motion_control_of_a_biped_robot_using_2_dof_gaze_control_structure

\section{INTECH}

open science | open minds

\author{
InTech Europe \\ University Campus STeP Ri \\ Slavka Krautzeka 83/A \\ 51000 Rijeka, Croatia \\ Phone: +385 (51) 770447 \\ Fax: +385 (51) 686166 \\ www.intechopen.com
}

\author{
InTech China \\ Unit 405, Office Block, Hotel Equatorial Shanghai \\ No.65, Yan An Road (West), Shanghai, 200040, China \\ 中国上海市延安西路65号上海国际贵都大饭店办公楼 405 单元 \\ Phone: +86-21-62489820 \\ Fax: +86-21-62489821
}


(C) 2007 The Author(s). Licensee IntechOpen. This chapter is distributed under the terms of the Creative Commons Attribution-NonCommercial-ShareAlike-3.0 License, which permits use, distribution and reproduction for non-commercial purposes, provided the original is properly cited and derivative works building on this content are distributed under the same license. 